\title{
Die Altersteilzeit im Zusammenspiel individueller und betrieblicher Einflussfaktoren
}

Susanne Wanger

Die Altersteilzeit hat sich in den vergangenen Jahren zu einem beliebten Modell für den Übergang vom Arbeitsleben in den Ruhestand entwickelt. Ende 2008 befand sich jeder sechste der 55- bis 64-jährigen Beschäftigten in Altersteilzeit. Zwar ist die Förderung der Altersteilzeit Ende 2009 ausgelaufen, ohne Zuschüsse wird Altersteilzeit jedoch auch weiterhin möglich sein, wenn entsprechende tarifliche bzw. betriebliche Regelungen getroffen oder erneuert werden. Der Beitrag untersucht - differenziert nach Geschlecht und Region -, welche Beschäftigtengruppen in der jüngeren Vergangenheit besonders von Altersteilzeitvereinbarungen profitiert haben.

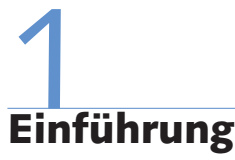

Geregelt wird die Altersteilzeit durch das 1996 in Kraft getretene Altersteilzeitgesetz (Übersicht 1). Das Altersteilzeitgesetz sollte - so die Intention - älteren Arbeitnehmern den gleitenden Übergang vom Erwerbsleben in den Ruhestand ermöglichen, um die Arbeitsbelastungen im Alter zu reduzieren und einen „Ruhestandsschock“ $\mathrm{zu}$ vermeiden. Außerdem wollte man so der zunehmenden Frühverrentungspraxis vorangegangener Jahre begegnen. ${ }^{1}$ Auch der Wissenstransfer zwischen den Generationen und die Vermeidung von Arbeitslosigkeit war ein Ziel: Arbeitsplätze sollten frei werden, um Arbeitslose zu integrieren, Auszubildende einzustellen oder Ausgebildete zu übernehmen. Denn nur in diesen Fällen wurde die Altersteilzeit von der Bundesagentur für Arbeit (BA) durch Zuschüsse gefördert. Diese Fördermöglichkeit ist Ende des Jahres 2009 für Neueintritte ausgelaufen.

Allerdings: Diese Zielsetzungen wurden nur teilweise verwirklicht. So ist die Altersteilzeit in der Regel nicht das, was ihr Name verspricht - sie ist meist keine Teilzeit und sie führt auch nur selten gleitend in den Ruhestand. Fast immer wurde und wird sie zu einem übergangslosen vorzeitigen Ausstieg aus dem Erwerbsleben genutzt (Blockmodell). So hat in den letzten Jahren der hohe Anteil des Blockmodells weiter zugenommen - bis Ende Dezember 2009 stieg er auf rd. $90 \% .^{2}$ Auch wird nur rund ein Drittel aller Beschäftigten in

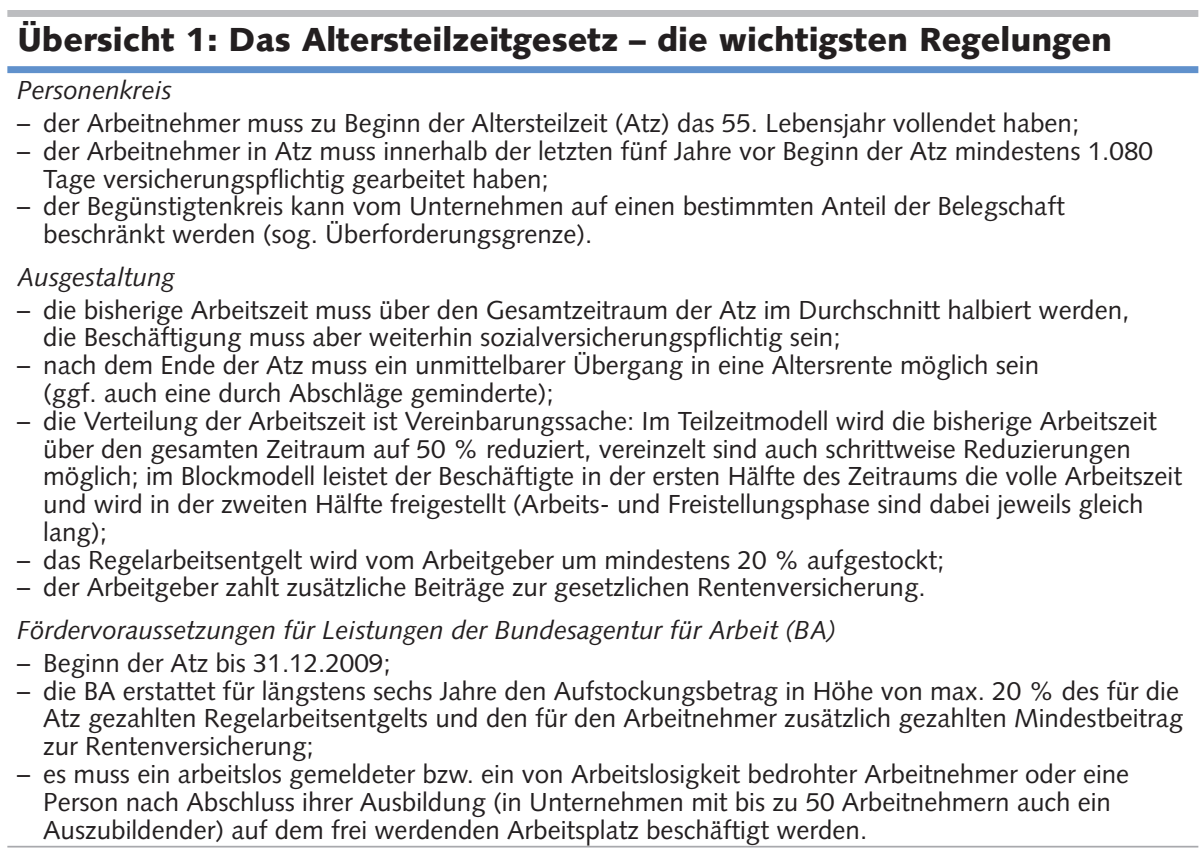

Quelle: Altersteilzeitgesetz (AtG), vgl. auch Wanger 2009. WSI MITTEILUNGEN

Altersteilzeit durch die BA gefördert, d.h. ihre frei gewordenen Arbeitsplätze wurden wiederbesetzt (Wanger 2009). Ende 2009 geschah dies in $38 \%$ der Fälle mit Arbeitslosen, die Wiederbesetzung mit frisch Ausgebildeten und Auszubildenden lag bei $62 \%$. Ein Großteil der Unternehmen verzichtete auf die Förderung und nutzte die Altersteilzeit zum sozialverträglichen Personalabbau bzw. zur Verjüngung der Belegschaft.

Im Fokus dieses Beitrags steht die Frage, welche Faktoren für die Inanspruchnahme von Altersteilzeitregelungen verantwortlich gemacht werden können, insbesondere ob bestimmte Beschäftigtengruppen be-
Zum Entstehungskontext des Altersteilzeitgesetzes und weiteren Diskussionssträngen siehe auch Barkholdt (2001); Stück (2003); Lindecke et al. (2007) sowie Knuth (2010).

2 Diese Zahlen gelten für die durch die BA geförderten Altersteilzeit-Arbeitsverhältnisse. Verschiedene Erhebungen (Ellguth/Koller 2000; Klammer/ Weber 2001; Hofmann 2008) zeigen, dass der Anteil des Blockmodells auch bei der ungeförderten Altersteilzeit ähnlich hoch sein dürfte.

Susanne Wanger, wissenschaftliche Mitarbeiterin der Forschungsgruppe Arbeitszeit und Arbeitsmarkt am Institut für Arbeits markt- und Berufsforschung (IAB), Nürnberg. Arbeitsschwerpunkte: Arbeitszeitforschung. e-mail: Susanne.Wanger@iab.de 
günstigt wurden. Zunächst geben wir einen Überblick über die bisherige Entwicklung wie auch die weiteren Perspektiven der Altersteilzeit (Abschnitt 2). AnschlieBend wird der neuere Forschungsstand zur Altersteilzeit in Deutschland skizziert (Abschnitt 3). Abschnitt 4 formuliert Hypothesen zum Einfluss individueller, betrieblicher und struktureller Merkmale auf die Inanspruchnahme von Altersteilzeit, die anschließend empirisch geprüft werden (Abschnitt 6). Die entsprechende Datenbasis erläutert vorab Abschnitt 5. Der Beitrag schließt mit einer Zusammenfassung der wichtigsten Resultate (Abschnitt 7).

\section{Entwicklung und Perspek- tiven der Altersteilzeit}

Ende 2008 befanden sich insgesamt rund 530.000 Personen im Alter zwischen 55 und 64 Jahren in einer Altersteilzeitbeschäftigung - gefördert sowie nicht gefördert. Nach einer kontinuierlichen Zunahme bis Anfang 2007 stabilisierte sich ihre Zahl bis Ende 2008 auf dem erreichten Niveau (Abbildung 1). Seit Mitte des Jahres 2004 nahm zugleich die reguläre sozialversicherungspflichtige Beschäftigung der Älteren insgesamt wieder zu (ohne Altersteilzeit). Ihre verbesserte Arbeitsmarktsituation war nicht allein konjunkturell bedingt, denn die sozialversicherungspflichtige Beschäftigung im Ganzen ging bis ins Jahr 2006 zurück. Entgegen diesem generellen Trend ist die Zahl der älteren Beschäftigten in den vergangenen Jahren gestiegen, weil geburtenstarke Jahrgänge in die höheren Altersgruppen aufgerückt sind. Gesetzliche Änderungen, die Einschränkung der Frühverrentung und Arbeitsmarktreformen der vergangenen Jahre spielten zusätzlich eine wichtige Rolle (Arlt et al. 2009).

Vor diesem Hintergrund veränderte sich der proportionale Anteil der Altersteilzeit. Von allen Beschäftigten in der anspruchsberechtigten Altersgruppe der 55- bis 64-Jährigen befand sich Ende 2008 jeder sechste in Altersteilzeit, bei den 60 - bis 64-Jährigen sogar jeder vierte. Bis Anfang 2006 war dieser Anteil kontinuierlich gestiegen, danach nahm er leicht ab. So hat wohl die kräftige Nachfrage nach qualifizierten Arbeitskräften im konjunkturellen Aufschwung der Jahre 2006/2007 den Einsatz von Altersteilzeit verringert.

\section{Abb. 1: Entwicklung der Beschäftigung Älterer}

Altersteilzeit-Beschäftigte (ATZ) sowie regulär sozialversicherungspflichtig Beschäftigte (SVB) ohne ATZ zwischen 55 und 64 Jahren - Personen in Tsd.* -

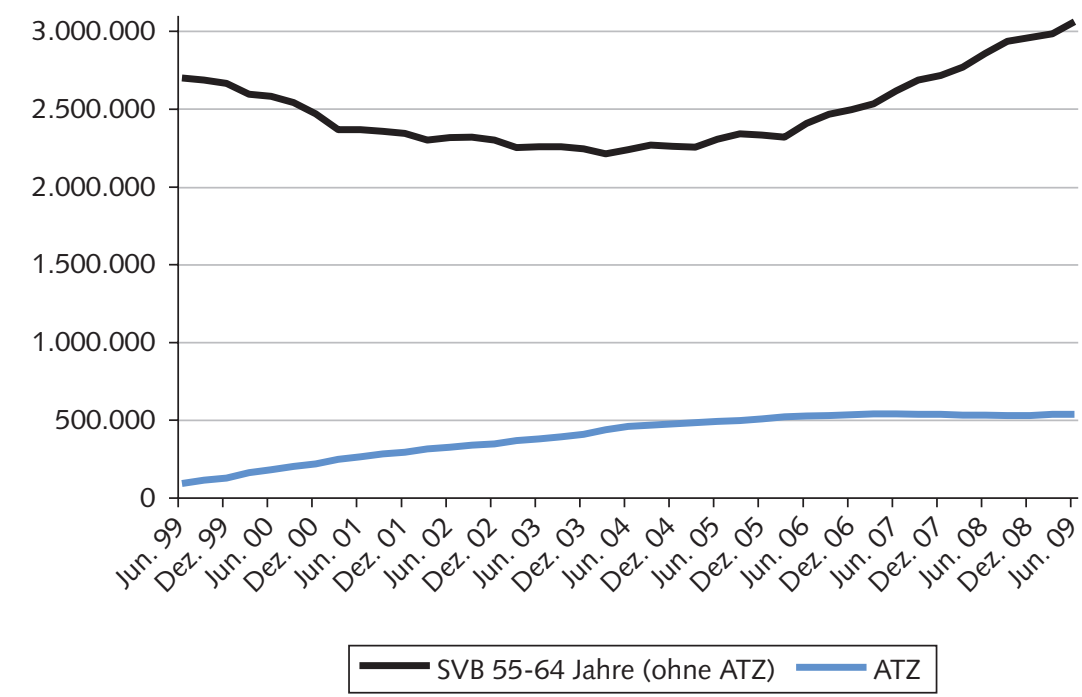

*jeweils Bestand am Quartalsende.

Quelle: IAB-Beschäftigtenhistorik; Bundesagentur für Arbeit; Darstellung der Autorin.

WSI MitTEILUNGEN
Die Inanspruchnahme der Altersteilzeit scheint-gemessen an ihrem Anteil an der Beschäftigung Älterer insgesamt - damit ihren Höhepunkt überschritten zu haben. Aber vermutlich wurde die Altersteilzeit im zweiten Halbjahr 2009 (Daten hierzu liegen noch nicht vor) aufgrund der Folgen der Finanzkrise wieder vermehrt von Betrieben eingesetzt, um Personal sozialverträglich abzubauen. Zusätzlich kann das Auslaufen der Regelung Ende 2009 zu Vorzieheffekten geführt haben, weil Beschäftigte und Betriebe die geförderte Altersteilzeit noch nutzen wollten.

Doch obgleich die Förderung der Altersteilzeit durch die BA für Neueintritte Ende 2009 ausgelaufen ist, bleibt das Gesetz an sich gültig, sodass nicht geförderte Altersteilzeit auch weiterhin möglich sein wird.

Die Altersteilzeit wird folglich nicht zwangsläufig aus der Personalpolitik der Betriebe verschwinden. Maßgeblich für den Abschluss von Altersteilzeitvereinbarungen sind die betrieblichen und tarifvertraglichen Regelungen der jeweiligen Branche, denn ein Rechtsanspruch auf Altersteilzeit besteht nicht. Das Altersteilzeitgesetz legt nur die Mindeststandards für Bedingungen und Leistungen fest, die weitere Ausgestaltung obliegt den Tarifvertragsparteien. Für die Arbeitnehmer verbesserte die tarifliche Ausgestaltung des gesetzlichen Rahmens bisher häufig die Bedingungen. Dazu gehörten etwa eine höhere Aufstockung des Teilzeitentgelts oder der Beiträge zur Rentenversicherung durch den Arbeitgeber. Schätzungsweise arbeiten zwei Drittel aller sozialversicherungspflichtig Beschäftigten in Betrieben, die tarifvertraglich oder durch Betriebsvereinbarung die Möglichkeit zur Altersteilzeit haben (Brussig et al. 2009). Die meisten Tarifverträge sind zum 31. Dezember 2009 ausgelaufen, dem letztmöglichen Eintrittszeitpunkt in die geförderte Altersteilzeit. Einige Branchen haben ihre Tarifverträge zur Altersteilzeit über das Jahr 2009 hinausgehend verlängert - zumeist auf Basis der bisherigen finanziellen Konditionen, jedoch häufig mit eingeschränktem Begünstigtenkreis. Vielfach handelt es sich hier um Branchen, die bereits vor 2010 die Altersteilzeit rege genutzt hatten, wie z.B. das Bank- und Versicherungsgewerbe, der Öffentliche Dienst sowie Teile des Produzierenden Gewerbes. Im Geltungsbereich dieser neuen Altersteilzeit-Tarifverträge arbeiten insgesamt rund 6,4 Mio. Arbeitnehmer. $^{3}$

3 Auswertungen auf Basis der Tarifpolitischen Informationen (bis 5/2010) des WSI-Tarifarchivs. 


\section{Forschungsstand zur Altersteilzeit}

Die Entscheidung für den Renteneintritt ist eng damit verbunden, welche Wege in den Ruhestand den Betroffenen offenstehen, d.h. aus welcher Erwerbs- aber auch Nichterwerbsform heraus gewechselt wird (Hoffmann 2007). Wie der Austritt aus dem Erwerbsleben gestaltet wird, hängt neben institutionellen und strukturellen Voraussetzungen auch von individuellen Gegebenheiten und den persönlichen Präferenzen ab (Arnds/Bonin 2002; Himmelreicher et al. 2008). Dieser Beitrag befasst sich mit einem spezifischen Pfad in den Ruhestand, welcher vielen älteren Beschäftigten seit 1996 offensteht, der Altersteilzeit. Sie ermöglicht im Grunde zwei alternative Wege des Eintritts in den Ruhestand: über das Teilzeitmodell kann ein gleitender Übergang realisiert werden, das Blockmodell ermöglicht einen vorzeitigen Ausstieg aus dem Erwerbsleben über die dem Renteneintritt vorgelagerte Freistellungsphase. Da der Großteil im Blockmodell ausgestaltet ist, wird die Altersteilzeit überwiegend als Weg zur Frühausgliederung genutzt. 2008 erfolgten 14,8 \% (2001: 6,8 \%) der Rentenzugänge aus Altersteilzeitbeschäftigungen (DRV 2009). Der steigende Anteil der Altersteilzeit an den Rentenzugängen ging mit einem Rückgang des Anteils der vorher „regulär“ versicherungspflichtig Beschäftigten sowie der Leistungsempfänger nach dem SGB III einher. So wurde in den letzten Jahren versicherungspflichtige Beschäftigung, aber auch Arbeitslosigkeit, teilweise durch Altersteilzeit substituiert (Hoffmann 2007).

\subsection{PUSH- UND PULL-ANSÄTZE}

Für die Erklärung des Erwerbsaustrittsbzw. Renteneintrittszeitpunktes werden in der wissenschaftlichen Literatur zumeist zwei konkurrierende mikroökonomische Erklärungsansätze unterschieden: Auf der einen Seite stehen negative Faktoren, die ältere Beschäftigte aus dem Erwerbsleben herausdrängen bzw. sie drängen, es zu beenden („push“). Solche Push-Faktoren können ein schlechter Gesundheitszustand, Arbeitslosigkeit oder Defizite in der beruflichen Qualifikation sein, wenn diese nicht mehr den aktuellen Anforde- rungen entspricht. Diese Ansätze betonen die strukturellen Bedingungen für die Beendigung der Erwerbsphase. Als maßgebliche Instanz werden die Betriebe angesehen, die ein Verwertungsinteresse am Produktionsfaktor Arbeit haben. Die Anreizgestaltung des Sozialrechts ist eher zweitrangig, vorrangig wirksam ist der von der jeweiligen Beschäftigungs- und Arbeitsmarktlage ausgehende „Druck“ auf ältere Arbeitnehmer zum Ausscheiden aus dem Arbeitsmarkt (Hoffmann 2007).

Auf der anderen Seite gibt es Faktoren, die den Ruhestand für die Arbeitnehmer „anziehend“ machen bzw. sie motivieren, aus dem Erwerbsleben auszuscheiden („pull“). Das können positive Erwartungen an einen vorgezogenen Ruhestand bei ausreichender finanzieller Absicherung, die familiäre Situation, aber auch der Wunsch nach mehr Freizeit sein. In „Pull“-Erklärungsansätzen werden die Entscheidungen über den Zeitpunkt des Überganges in den Ruhestand hauptsächlich von individuellen Faktoren und von der Anreizwirkung des Sozialrechts bezüglich Renteneintrittsmöglichkeiten und Rentenhöhe bestimmt (Barkholdt 2001).

„Pull“-Ansätze unterstellen den Arbeitnehmern überwiegend die Initiative $\mathrm{zu}$ freiwillig angestrebtem Übergang in den Ruhestand, „Push “-Ansätze hingegen mehr ein Reagieren bzw. eher einen unfreiwilligen Rückzug aus dem Arbeitsmarkt. Die empirischen Befunde zur Altersteilzeit zeigen, dass die Faktoren, die für die Entscheidung, den Weg der Altersteilzeit zu beschreiten, ausschlaggebend sind, sich nicht eindeutig einem Ansatz zuordnen lassen. Vielmehr setzen sie sich aus einer Mischung von Push- und Pull-Faktoren zusammen.

\subsection{EMPIRISCHER WISSENSSTAND}

Arnds/Bonin (2002) werten die Regelungen zur Altersteilzeit als Ausdruck einer Arbeitsmarktpolitik, die ältere Arbeitnehmer als Belastung des Arbeitsmarktes versteht und daher einen längeren Verbleib im Erwerbsleben gar nicht anstrebt. Demnach hat die Altersteilzeit von vorneherein den vorzeitigen Eintritt in die Altersrente zum Ziel, der bis 2006 bereits mit 60 Jahren möglich war. ${ }^{4}$ Tatsächlich erfolgt der Ausstieg aus dem Erwerbsalltag meist viel früher: Infolge der verbreiteten Nutzung des Blockmodells und der dem Rentenbeginn vorgelagerten Freistellungsphase ar- beiten Altersteilzeitbeschäftigte im Schnitt bereits drei Jahre vor dem Renteneintritt nicht mehr (Radl 2007).

Zusätzlich führt Altersteilzeitbeschäftigung tendenziell früher in die Rente, versicherungspflichtige Beschäftigung ohne Altersteilzeit scheint dagegen die beste Basis für einen späteren Renteneintritt zu sein (Kaldybajewa/Kruse 2007). Auch aufgrund von rentenrechtlichen Regelungen gehen Altersteilzeitbeschäftigte im Durchschnitt zwei Jahre früher in Rente als die anderen sozialversicherungspflichtig Beschäftigten (Radl 2006). Hoffmann (2007) berechnet, dass das Verrentungsrisiko für Altersteilzeitbeschäftigte dreieinhalb mal höher ist als für andere sozialversicherungspflichtige Beschäftigte. Altersteilzeitbeschäftigte gehen außerdem weitestgehend aus freien Stücken und mit guter finanzieller Abfederung aus dem Berufsleben (Radl 2007). Denn sie erreichen eine ausgeprägt überdurchschnittliche Entgeltposition (Hoffmann 2007). So spricht - gemäß dem „Pull“-Argument - vieles dafür, dass Altersteilzeitbeschäftigte häufig Personenkreisen mit hoher Freizeitpräferenz zuzuordnen sind, die sich einen vorgezogenen Rentenzugang unter Inkaufnahme von Abschlägen leisten. ${ }^{5}$ Die Inanspruchnahme konzentrierte sich, insbesondere in den ersten Jahren nach Einführung der Altersteilzeit, weitgehend auf Männer aus den alten Bundesländern (Kaldybajewa 2004). Nach der Ausweitung des Berechtigtenkreises auf teilzeitbeschäftigte Personen (ab dem Jahr 2000) ist vor allem der Anteil westdeutscher Frauen an den Altersteilzeitbeschäftigten gestiegen (Wanger 2009). Ehemalige Altersteilzeitbeschäftigte beziehen außerdem mit die höchsten durchschnittlichen Altersrenten (DRV 2009). So sind die Biografien von Altersteilzeitbeschäftigten typischerweise von längeren Versicherungszeiträumen, höheren Entgeltpositionen und deutlich kürzeren Arbeitslosigkeitszeiten geprägt als bei Personen, die aus Arbeitslosigkeit und anderen versicherungspflichtigen Beschäftigungen in den Ruhestand wechseln.

$4 \quad$ In der Regel ist die Altersteilzeitarbeit mit einem vorgezogenen Renteneintritt verbunden, da die Altersrente nach Altersteilzeitarbeit bislang noch eine vorzeitige, mit Abschlägen reduzierte Rente ermöglicht. Zu den genauen Regelungen siehe Hirschenauer (2007); Wanger (2009).

52008 mussten $98 \%$ der Rentenzugänge aus Arbeitslosigkeit bzw. Altersteilzeit Abschläge in Kauf nehmen (DRV 2009). 
Altersteilzeitbeschäftigte erweisen sich also insgesamt als eine nach sozialen Kriterien relativ starke Gruppe (Kaldybajewa/ Kruse 2007).

Neben der Einkommenssituation wird vor allem der beruflichen Tätigkeit eine hohe Bedeutung für die Wahl des Verrentungspfades zugeschrieben (Radl 2006). So profitieren vor allem Angestellte in Büroberufen von Altersteilzeitregelungen (Stück 2003; Wanger 2009). Insgesamt zeigt die Betrachtung der Inanspruchnahme von Altersteilzeit kein Muster, das auf einen gezielten Einsatz in Berufen oder Branchen mit hohen Belastungen schließen lässt (Wanger 2009). Allerdings sehen die meisten tarifvertraglichen Regelungen auch kaum Bestimmungen vor, die zu einem solchen zielgerichteten Einsatz hätte führen können (Brussig et al. 2009).

Vereinbarungen zur Altersteilzeit sind im öffentlichen Sektor weiter verbreitet als in der Privatwirtschaft (Klammer/Weber 2001). Auch eine Studie auf europäischer Ebene (Leber/Wagner 2007) zeigt, dass es vor allem vom Wirtschaftszweig abhängt, ob in einem Unternehmen Altersteilzeitregelungen angeboten werden. So gibt es z. B. in Unternehmen aus den Branchen Kredit- und Versicherungsgewerbe, Erziehung und Unterricht sowie Gesundheits-/Veterinär- und Sozialwesen häufiger Altersteilzeitregelungen; ebenso in Unternehmen, die gute Erfahrungen mit Teilzeitarbeit und flexibler Arbeitszeitgestaltung gemacht haben. Außerdem besteht ein enger Zusammenhang zwischen Altersteilzeitarbeit und Betriebsgröße: In größeren Betrieben ist sie öfter zu finden. Ergebnisse des IAB-Betriebspanels (Ellguth/Koller 2000) belegen, dass das betriebliche Engagement in Sachen Altersteilzeit mit der Betriebsgröße zunimmt. Insbesondere größere Betriebe nutzen die Altersteilzeit offensichtlich als einen Weg, die Belegschaft zu verjüngen. So gehen die Beschäftigten in den Großbetrieben sowohl früher als in den anderen Betrieben in die Altersteilzeit als auch jünger in die Altersrente (Wanger 2009).

Zusammenfassend zeigt der Forschungsstand, dass bestimmte Beschäftigtengruppen häufiger in Altersteilzeit zu finden sind. Sie wird vielfach zu einem vorzeitigen Ausstieg aus dem Erwerbsleben genutzt und der tarifvertraglichen Ausgestaltung kommt ein hoher Stellenwert zu. Neben den vorgestellten, meist deskriptiven Forschungsarbeiten liegen bisher keine multivariaten Analysen zur Altersteilzeit vor, die den Einfluss und das Zusammenwirken mehrerer Bestimmungsgrößen auf die Nutzung systematisch untersuchen. Die im Folgenden präsentierten Auswertungen sollen dazu beitragen, diese Lücke zu schließen.

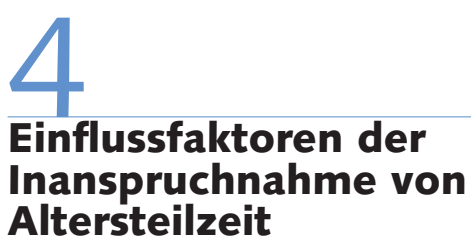

Welche individuellen Faktoren können einen Einfluss auf die Inanspruchnahme von Altersteilzeitregelungen haben? Welche Rolle spielen dabei Charakteristika des Beschäftigungsbetriebes bzw. der Beschäftigungsbranche und die strukturellen Rahmenbedingungen? Zur Beantwortung dieser Fragen bilden wir Hypothesen, die relevant für die Inanspruchnahme von Altersteilzeit sein dürften, und unterscheiden dabei nach individuellen, betrieblichen sowie strukturellen Faktoren.

\subsection{INDIVIDUELLE FAKTOREN}

Für Personen mit höherem Einkommen dürfte - entsprechend den Pull-Ansätzen - die Altersteilzeit attraktiver sein. Denn das reduzierte Einkommen während der Altersteilzeitarbeit sowie eventuell später anfallende Rentenabschläge muss der Beschäftigte sich auch leisten können. Aus diesen Gründen ist zu vermuten, dass die Wahrscheinlichkeit der Inanspruchnahme von Altersteilzeit mit steigendem Einkommen zunimmt (Hypothese 1). Höheres Einkommen geht in der Regel mit höherer beruflicher Qualifikation einher, deshalb dürften gut Ausgebildete häufiger in Altersteilzeitbeschäftigungen zu finden sein. Mit steigendem Qualifikationsniveau dürften die Chancen auf Altersteilzeit aber wieder sinken (Hypothese 2). Denn Beschäftigte mit höherer Bildung haben aus Sicht der Push-Ansätze ein niedrigeres Renteneintrittsrisiko, weil ihre Beschäftigungschancen im Alter höher sind (Radl 2007). Da hohe Qualifikation häufig mit hohem Einkommen einhergeht, kann die beim Verbleib im Erwerbsleben erwartbare höhere Rente das Risiko eines vorzeitigen Renteneintritts (Arnds/Bonin 2002) verringern. Außerdem sind höher qualifizierte Erwerbspersonen während ihrer
Berufsbiografie geringeren gesundheitlichen Risiken ausgesetzt und verbleiben so deutlich häufiger als gering Qualifizierte länger im Erwerbsleben (Himmelreicher et al. 2008).

Ein Ziel des Altersteilzeitgesetzes war es, dem zum Teil eingeschränkten Leistungsvermögen von älteren Beschäftigten gerecht zu werden, denn nicht jede Berufstätigkeit kann ohne Weiteres in vollem Umfang bis ins hohe Alter ausgeübt werden. So kann ein schlechter Gesundheitszustand das Interesse an einem vorzeitigen Ausstieg aus dem Erwerbsleben über die Altersteilzeit erhöhen. Dies spricht dafür, dass in Berufen, die durch hohe körperliche Belastungen oder durch andere hohe Arbeitsanforderungen gekennzeichnet sind, die Inanspruchnahme von Altersteilzeit höher sein dürfte (Hypothese 3).

Von Bedeutung für die Inanspruchnahme von Altersteilzeit könnte auch die Staatsangehörigkeit des Beschäftigten sein. Ein Großteil der Arbeitsmarktnachteile von Ausländern kann zwar über Unterschiede im formal erreichten Ausbildungsabschluss erklärt werden, daneben könnten aber auch unvollständige Erwerbsbiografien sowie Informationsdefizite aufgrund von Sprachbarrieren eine Rolle spielen. Deshalb dürften Ausländer eine geringere Wahrscheinlichkeit als Deutsche haben, in Altersteilzeit zu sein (Hypothese 4).

Altersteilzeit kann ab dem 55. Lebensjahr vereinbart werden. Im Jahr 2007 waren Altersteilzeitbeschäftigte bei Eintritt durchschnittlich 57,7 Jahre und bei Austritt 61,9 Jahre alt. Das Austrittsalter ist in den vergangenen Jahren zwar gestiegen (Wanger 2009), aber noch immer führt Altersteilzeitbeschäftigung tendenziell früher zu einem Renteneintritt als reguläre sozialversicherungspflichtige $\mathrm{Be}$ schäftigung (Abschnitt 2). Deshalb dürfte die Wahrscheinlichkeit auf Altersteilzeit in der Altersgruppe bis 58 Jahren ebenso wie in den Jahren vor dem regulärem Rentenbeginn (ab 63 Jahre) geringer sein (Hypothese 5).

Weiterhin könnte auch die Wochenarbeitszeit einen Einfluss auf die Inanspruchnahme von Altersteilzeit haben. Da das Beschäftigungsverhältnis während der Altersteilzeit weiterhin sozialversicherungspflichtig sein muss, könnten bestimmte Gruppen von Teilzeitbeschäftigten bei einer weiteren Reduzierung ihrer Arbeitszeit dieses Kriterium nicht mehr erfüllen und 
von einer Inanspruchnahme von vornherein ausgeschlossen sein. Teilzeitbeschäftigte dürften somit eine geringere Chance auf Altersteilzeit haben als Vollzeitbeschäftigte (Hypothese 6).

\subsection{BETRIEBLICHE FAKTOREN}

Daneben können auch betriebliche Einflussfaktoren die Inanspruchnahme von Altersteilzeit bestimmen. Kleinere Betriebe dürften vermehrt darauf angewiesen sein, erfahrene und damit schwer zu ersetzende Arbeitskräfte möglichst lange im Betrieb zu halten. Die großen Betriebe haben dagegen mehr organisatorische und personalpolitische Möglichkeiten, um den reibungslosen Betriebsablauf bei Altersteilzeit aufrechtzuerhalten. In Kleinbetrieben könnte eine geringere Flexibilität möglicherweise zu finanziellen Einschränkungen führen, sodass sie die Kosten der Vereinbarungen zur Altersteilzeit nicht tragen können. So dürfte in größeren Betrieben die Wahrscheinlichkeit auf Altersteilzeit höher sein als in kleineren (Hypothese 7). In Großbetrieben können außerdem Betriebsräte die Umsetzung von Altersteilzeitregelungen fördern. Zu erwarten ist, dass insbesondere in Branchen mit hoher Tarifbindung viele Altersteilzeitbeschäftigte zu finden sind, da für den Anspruch auf Altersteilzeit ein entsprechender Tarifvertrag vorliegen muss (Hypothese 8). Darauf deuten auch Studien zu Frühverrentungen allgemein hin, die häufiger und in größerem Ausmaß in tarifgebundenen Betrieben stattfinden (Bellmann/Janik 2009).

Weiterhin dürfte in Wirtschaftszweigen, wo die Tarifvertragsparteien besonders günstige Altersteilzeitregelungen ausgehandelt haben - z.B. über die gesetzlichen Regelungen hinausgehende Aufstockungsbeträge -, die Inanspruchnahme attraktiver sein (Hypothese 9). Ein auf Branchenebene überdurchschnittlicher Anteil von Geförderten an allen Altersteilzeitbeschäftigungen könnte ein Indiz dafür sein, dass in diesen Branchen die Inanspruchnahme allgemein auch höher ist (Hypothese 10).

Von weiterer Bedeutung könnte die Beschäftigungsentwicklung der Branche sein, z.B. weil schrumpfende Betriebe oder Branchen aufgrund konjunktureller oder struktureller Einbrüche die Altersteilzeit zur personalpolitischen Selektion bzw. zur Verjüngung der Belegschaft nutzen. Negative Beschäftigungsentwicklungen könnten dann mit einer höheren Inanspruchnahme von Altersteilzeitregelungen einhergehen (Hypothese 11).

\subsection{STRUKTURELLE FAKTOREN}

Neben den individuellen und betrieblichen Merkmalen können auch verschiedene Umfeldvariablen eine Rolle spielen. So könnte die regionale Arbeitsmarktlage bzw. -anspannung einen Einfluss auf die Inanspruchnahme von Altersteilzeitregelungen haben. Ein Indikator für die Stärke der Arbeitskräftenachfrage ist die Vakanzrate, das Verhältnis zwischen Vakanzen und aktuell Beschäftigten. Sind viele Arbeitsplätze vakant, ist der Druck für Ältere, in den Ruhestand zu gehen, geringer und die Wahrscheinlichkeit auf Altersteilzeit möglicherweise kleiner (Hypothese 12). Ein weiterer Indikator ist die regionale Arbeitslosigkeit. So könnten hohe Arbeitslosenquoten dazu führen, dass Ältere häufiger über Altersteilzeit aus dem Erwerbsleben ausscheiden, um Arbeitsplätze für Jüngere freizumachen (Hypothese 13).

Weitere Einflussfaktoren, insbesondere solche, die im Zusammenhang mit dem Haushaltskontext der Beschäftigten stehen, können mangels entsprechender Daten in der Analyse nicht berücksichtigt werden. Deshalb werden sie hier nicht diskutiert.

\section{Datenbasis}

Für die vorliegende Untersuchung wurde eine Stichprobe aus der BeschäftigtenHistorik (BeH) des Instituts für Arbeitsmarkt- und Berufsforschung (IAB) verwendet. Die Grundlage der BeH bildet die Beschäftigtenstatistik der BA, die regelmäßige Meldungen der Arbeitgeber zu ihren sozialversicherungspflichtig Beschäftigten an die Sozialversicherungsträger enthält. Neben Informationen zu den Beschäftigten beinhaltet diese Datenbasis auch Angaben zu dem Betrieb. Die Stichprobe deckt insgesamt einen Zeitraum von 13 Jahren ab (01.01.1996-31.12.2008) und liefert somit auch Informationen zur Erwerbstätigkeit vor der Altersteilzeit. Ergänzt wurden diese Daten durch Informationen aus dem IABBetriebspanel, Daten aus den Geschäftsprozessen der BA sowie Ergebnisse der $\mathrm{BIBB} / \mathrm{BAuA}$-Erwerbstätigenbefragung
2005/2006. Diese wird gemeinsam vom Bundesinstitut für Berufsbildung (BIBB) sowie von der Bundesanstalt für Arbeitsschutz und Arbeitsmedizin (BAuA) durchgeführt.

Die Analyse betrifft die zum Stichtag 30.06.2007 beschäftigten Personengruppen „sozialversicherungspflichtig Beschäftigte ohne besondere Merkmale“ und „Altersteilzeitbeschäftigte" im Alter zwischen 55 und 64 Jahren. Insgesamt umfasst die Stichprobe fast 3 Mio. sozialversicherungspflichtig Beschäftigte, davon war etwa ein Fünftel altersteilzeitbeschäftigt. Weitere Personengruppen, wie Auszubildende, Hausgewerbetreibende, Praktikanten, Werkstudenten oder behinderte Menschen, spielen in der betrachteten Altersgruppe zahlenmäßig nur eine untergeordnete Rolle und gehen deshalb nicht in die Analyse ein.

Einige Variablen mussten für die Verwendung im Regressionsmodell erst umgeformt, andere mit externen Datensätzen verknüpft werden. So enthält die $\mathrm{BeH}$ die Tagesentgelte der Beschäftigten in Euro. Wir verwenden jedoch nicht das absolute Einkommen, sondern die relative Position des Beschäftigten im Vergleich zu den anderen sozialversicherungspflichtig Beschäftigten. Dazu teilen wir die Tagesentgelte der regulär sozialversicherungspflichtig Beschäftigten - getrennt für Teilzeit und Vollzeit - zum Untersuchungszeitpunkt in fünf Einkommensklassen (Quintile) ein. Den Altersteilzeitbeschäftigten wird die Einkommensklasse zum Zeitpunkt der letzten regulären Beschäftigung vor der Altersteilzeit zugeordnet; auch hier jeweils in Relation zu den regulär sozialversicherungspflichtig Beschäftigten. Dieses Vorgehen ermöglicht, dass Verdienste unterschiedlicher Zeitpunkte in die Analyse einbezogen werden können.

Die BeH enthält keine Informationen zu dem Gesundheitszustand eines Beschäftigten, deshalb greifen wir hilfsweise auf Ergebnisse einer Erwerbstätigenbefragung zu Arbeitsbedingungen und -belastungen zurück und verknüpfen diese über die Berufsgruppe mit den IAB-Daten (Wanger 2009). Zwei Belastungsindikatoren werden so gebildet und gehen als Dummys in das Modell ein. Der erste misst hohe körperliche Belastungen im ausgeübten Beruf. Der zweite Indikator misst den „Stressfaktor“, d.h. Arbeitsanforderungen, die im jeweiligen Beruf als belastend empfunden werden. 
Die Information zu der branchenspezifischen Tarifbindung stammt aus dem IAB-Betriebspanel (Ellguth/Kohaut 2008). Die Ergebnisse zu der Tarifbindung der Beschäftigten in den einzelnen Wirtschaftszweigen werden unserem Datensatz über die Branche zugeordnet - getrennt für West- und Ostdeutschland. Die Angaben, ob Branchen eine höhere Einkommensaufstockung als im Gesetz vorgesehen zahlen, stammen aus dem WSI-Tarifarchiv. Dazu haben wir die tarifvertraglichen Regelungen von über 100 Branchen ausgewertet und mittels der Wirtschaftszweig- und Arbeitsortvariablen unserem Datensatz zugespielt. Die Angaben zu den geförderten Altersteilzeitbeschäftigten wurden der Leistungsstatistik der BA entnommen. Die Vakanz- und Arbeitslosigkeitsraten stammen aus Geschäftsstatistiken der BA und werden über die Variable zur Kreisebene unserem Datensatz zugeführt.

Da auf Betriebsebene keine Informationen zur Beschäftigungsentwicklung für die in die Analyse eingehenden Betriebe vorliegen, nehmen wir dafür hilfsweise die Beschäftigungsentwicklung auf Wirtschaftsabschnittsebene. Dazu wird der Mittelwert der jahresdurchschnittlichen Veränderungen der Branchen zwischen den Jahren 2003 bis 2007 berechnet und ein Dummy für Branchen mit negativer Beschäftigungsentwicklung gebildet.

\section{Ergebnisse der ökono- metrischen Analyse}

Für die ökonometrische Analyse betrachten wir die Gruppe der 55- bis 64-jährigen sozialversicherungspflichtig Beschäftigten. Geschätzt wird ein Logit-Modell, dessen abhängige Variable den Wert eins annimmt, wenn der Beschäftigte sich am 30.06.2007 in Altersteilzeit befand und den Wert null, wenn der Ältere regulär sozialversicherungspflichtig beschäftigt war. Logit-Modelle liefern Erkenntnisse, ob deskriptiv gezeigte Zusammenhänge auch dann signifikant sind, wenn der Einfluss für alle verfügbaren Variablen kontrolliert wird. So kann Signifikanz, Einflussrichtung und Einflussstärke festgestellt werden. ${ }^{6}$ In Tabelle 1 sind die Ergebnisse dieses Logit-Modells zusammengefasst. Die hier ausgewiesenen Odds-Ratios sind als relative Chancenverhältnisse zu interpretieren.
Eine Odds-Ratio größer als eins zeigt an, dass die Chance auf Altersteilzeit höher ist als für die Referenzkategorie, bei kleiner als eins ist sie niedriger.

Die Bestimmungsgründe für die Altersteilzeit können sich aufgrund der unterschiedlichen regionalen Arbeitsmarktlage, aber auch wegen geschlechtsspezifischer Lebensläufe, unterscheiden. Deshalb führen wir die Schätzungen für vier Gruppen durch: für Männer und Frauen in West- und Ostdeutschland.

Betrachtet man die individuellen Faktoren, wird deutlich, dass - entsprechend der Hypothese 1 - Arbeitnehmer aus den unteren Einkommensquintilen eine signifikant geringere Chance auf Altersteilzeit haben. Beschäftigte mit einem niedrigeren Einkommen können sich eine Reduzierung des Einkommens sowie mögliche Rentenabschläge offensichtlich weniger leisten. Sie sind deshalb seltener in der Altersteilzeit zu finden - entsprechend der Sicht von Pull-Ansätzen. Bestätigt wird auch Hypothese 2, dass die berufliche Qualifikation einen signifikanten Einfluss hat. Für Beschäftigte mit Berufsausbildung ist die Aussicht auf Altersteilzeit höher als für solche ohne Berufsausbildung (bis auf Frauen in Westdeutschland), dagegen haben Beschäftigte mit Universitäts- und $\mathrm{FH}$-Abschluss eine geringere Wahrscheinlichkeit auf Altersteilzeitbeschäftigung.

Für die Belastungen im ausgeübten Beruf - einem häufig genannten Push-Argument - wird die Hypothese nur zum Teil bestätigt. So haben insbesondere ostdeutsche Männer, die in Berufen mit hohen körperlichen Belastungen arbeiten, sowie westdeutsche Frauen, die in Berufen mit belastenden Arbeitsanforderungen tätig sind, eine deutlich größere Wahrscheinlichkeit auf Altersteilzeit. Ansonsten haben insbesondere Berufsgruppen mit hohen körperlichen Belastungen eine geringere Wahrscheinlichkeit, in Altersteilzeit zu sein.

Hypothese 4, die einen starken Einfluss der Staatsangehörigkeit auf die Inanspruchnahme von Altersteilzeit vermutet, findet wiederum volle Bestätigung. Ausländer in Ostdeutschland und Beschäftigte aus dem nichteuropäischen Ausland werden dabei stärker benachteiligt. Inwieweit dies auf kürzere oder unvollständige Erwerbsbiografien oder eine direkte Diskriminierung im Betrieb zurückgeführt werden kann, lässt sich auf Basis der ver- wendeten Daten nicht beantworten. Auch die Hypothesen 5 und 6 in Bezug auf Alter und Arbeitszeitform werden anhand der logistischen Regression verifiziert.

Von statistisch signifikanter Bedeutung für die Inanspruchnahme sind zudem die Betriebsgröße sowie eine hohe Tarifbindung der Branche. Dieser Zusammenhang ist im Osten stärker ausgeprägt. Da der Zugang zur Altersteilzeit durch Tarifvertrag und betriebliche Vereinbarungen gesteuert wird, ist dieser starke Einfluss nicht verwunderlich. In Branchen, die ihren Beschäftigten höhere Aufstockungsleistungen als gesetzlich vorgeschrieben bieten, gibt es regionale Unterschiede: Während dies im Osten zu einer höheren Chance auf Altersteilzeit führt, ist im Westen das Gegenteil der Fall. Eventuell sind Beschäftigte im Osten aufgrund ihres niedrigeren Einkommens stärker als im Westen auf Aufstockungsleistungen angewiesen.

Werden in einer Branche häufig Altersteilzeitbeschäftigte gefördert, erhöht dies nicht unbedingt die Chance auf Altersteilzeit für die hier beschäftigten Älteren. So werden in Branchen, die in geringerem Ausmaß Altersteilzeit vereinbaren, häufiger Förderleistungen von der BA in Anspruch genommen und Stellen wiederbesetzt. Eventuell handelt es sich hier um Branchen, die Altersteilzeit nur dann vereinbaren, wenn die Aufstockungsbeträge auch ersetzt werden, d.h. wenn für sie die Altersteilzeit kostenneutral ist. Möglich ist auch, dass bestimmte Branchen die Altersteilzeit mit Wiederbesetzung als aktives

\footnotetext{
6 Wir haben die Stichprobe um die regulär Beschäftigten bereinigt, von denen uns bekannt war, dass sie nach dem Stichtag 30.06.2007 in die Altersteilzeit gewechselt sind. Jedoch könnten natürlich noch weitere ältere sozialversicherungspflichtig Beschäftigte - vor allem der jüngeren Jahrgänge - nach dem Ende des Stichprobenzeitraums (31.12.2008) in die Altersteilzeit wechseln. Das könnte sich auf die Schätzergebnisse auswirken und diese eventuell verzerren. Um dies zu überprüfen, haben wir das gleiche Modell zu einem früheren Stichtag (30.06.2002) in zwei Varianten durchgerechnet: In der ersten Berechnung haben wir die Stichtagszustände (SVB oder ATZ am 30.06.2002) verwendet. In einer zweiten Variante haben wir das Modell um die sozialversicherungspflichtig Beschäftigten bereinigt, von denen uns bekannt war, dass sie in den Folgejahren bis 2008 in die Altersteilzeit gewechselt sind. Die Erklärungsgüte des Modells (Pseudo $\mathrm{R}^{2}$ Mc Fadden) verbesserte sich durch die Bereinigung, Signifikanz und Einflussrichtung blieben erhalten, die Einflussstärke variierte jedoch. Die hohen Fallzahlen tragen z. T. zu der hohen Signifikanz des Modells bei.
} 
Tabelle 1: Logistische Regression - Abhängige Variable: Altersteilzeitbeschäftigt am Stichtag 30.06.2007

\begin{tabular}{|c|c|c|c|c|c|c|c|c|}
\hline \multirow{2}{*}{ Unabhängige Variablen } & \multicolumn{4}{|c|}{ West } & \multicolumn{4}{|c|}{ Ost } \\
\hline & \multicolumn{2}{|c|}{ Männer } & \multicolumn{2}{|c|}{ Frauen } & \multicolumn{2}{|c|}{ Männer } & \multicolumn{2}{|c|}{ Frauen } \\
\hline \multicolumn{9}{|l|}{ Individuelle Faktoren } \\
\hline \multicolumn{9}{|l|}{ Einkommen: (Referenz: 5. Quintil, höchste Einkommensklasse) } \\
\hline - 1. Quintil (unterste Einkommensklasse) & 0,14 & $* * *$ & 0,11 & $* * *$ & 0,05 & $* * *$ & 0,05 & $* * *$ \\
\hline - 2. Quintil & 0,29 & $* * *$ & 0,59 & $* * *$ & 0,45 & $* * *$ & 0,50 & *** \\
\hline - 3. Quintil (mittlere Einkommensklasse) & 0,72 & $* * *$ & 0,86 & $* * *$ & 0,75 & $* * *$ & 0,75 & *** \\
\hline - 4. Quintil & 0,94 & $* * *$ & 0,97 & $* * *$ & 1,14 & $* * *$ & 0,96 & \\
\hline \multicolumn{9}{|l|}{ Ausbildung (Referenz: ohne Berufsausbildung) } \\
\hline - FH-/Universitätsabschluss & 0,59 & $* * *$ & 0,45 & *** & 0,86 & *** & 1,04 & \\
\hline - mit Berufsausbildung & 1,12 & $* * *$ & 0,94 & $* * *$ & 1,10 & ** & 1,10 & *** \\
\hline - unbekannt & 0,40 & $* * *$ & 0,39 & $* * *$ & 0,48 & $* * *$ & 0,48 & *** \\
\hline Berufsgruppe mit hohen körperlichen Arbeitsbelastungen & 0,97 & $* * *$ & 0,76 & $* * *$ & 1,32 & $* * *$ & 0,86 & *** \\
\hline Berufsgruppe mit belastenden Arbeitsanforderungen & 1,07 & $* * *$ & 1,22 & $* * *$ & 0,91 & $* * *$ & 1,07 & $* * *$ \\
\hline \multicolumn{9}{|l|}{ Nationalität: (Referenz: Deutscher) } \\
\hline - Europäer & 0,83 & $* * *$ & 0,87 & $* * *$ & 0,68 & $* * *$ & 0,58 & *** \\
\hline - Sonstige & 0,49 & $* * *$ & 0,42 & $* * *$ & 0,32 & $* * *$ & 0,19 & $* * *$ \\
\hline \multicolumn{9}{|l|}{ Alter (Referenz: 63 Jahre und älter) } \\
\hline - 55 bis 58 Jahre & 0,31 & $* * *$ & 0,40 & $* * *$ & 0,27 & $* * *$ & 0,36 & $* * *$ \\
\hline - 59 bis 62 Jahre & 1,36 & $* * *$ & 1,53 & $* * *$ & 1,20 & $* * *$ & 1,59 & $* * *$ \\
\hline Arbeitszeitform: Teilzeit & 1,07 & $* * *$ & 0,95 & $* * *$ & 0,67 & $* * *$ & 0,54 & $* * *$ \\
\hline \multicolumn{9}{|l|}{ Betriebliche Faktoren } \\
\hline \multicolumn{9}{|l|}{ Betriebsgröße (Referenz: 500 und mehr Beschäftigte) } \\
\hline - 1 bis 49 SV-Beschäftigte & 0,20 & $* * *$ & 0,25 & $* * *$ & 0,44 & $* * *$ & 0,68 & $* * *$ \\
\hline - 50 bis 499 SV-Beschäftigte & 0,43 & $* * *$ & 0,52 & $* * *$ & 0,66 & $* * *$ & 0,91 & $* * *$ \\
\hline Branche mit hoher Tarifbindung der Beschäftigten & 1,39 & $* * *$ & 2,12 & $* * *$ & 2,27 & *** & 2,84 & *** \\
\hline Branche mit höheren Aufstockungsregelungen & 0,96 & $* * *$ & 0,95 & $* * *$ & 1,29 & $* * *$ & 1,31 & $* * *$ \\
\hline Branche mit hoher Wiederbesetzungsquote & 0,49 & $* * *$ & 0,55 & $* * *$ & 0,46 & $* * *$ & 0,46 & *** \\
\hline Negative Beschäftigungsentwicklung in der Branche & 1,10 & $* * *$ & 1,15 & $* * *$ & 0,81 & $* * *$ & 0,72 & $* * *$ \\
\hline \multicolumn{9}{|l|}{ Strukturelle Faktoren } \\
\hline Regionale Arbeitsmarktlage: hohe Vakanzrate & 0,91 & $* * *$ & 0,90 & $* * *$ & 1,20 & $* * *$ & 1,22 & $* * *$ \\
\hline Regionale Arbeitsmarktlage hohe Arbeitslosenquote & 1,07 & $* * *$ & 1,06 & $* * *$ & 1,29 & $* * *$ & 1,16 & $* * *$ \\
\hline N : gültige Fälle & 1.285 & 5.901 & 969.9 & 990 & 302.4 & 411 & 296.2 & \\
\hline Pseudo-R² (Mc Fadden) & 0,200 & & 0,222 & & 0,275 & & 0,308 & \\
\hline $\begin{array}{l}* / * * / * * * \text { Statistische Signifikanz auf dem 5/1/0,1-\% Niveau. } \\
\text { Quelle: IAB-Beschäftigtenhistorik; Berechnungen der Autorin. }\end{array}$ & & & & & & & & \\
\hline
\end{tabular}

Instrument zur Strukturverbesserung einsetzen.

Die Betrachtung der durchschnittlichen Branchenentwicklung zwischen den Jahren 2003 bis 2007 führt zu regional unterschiedlichen Ergebnissen: Im Westen war in Branchen mit abnehmender Beschäftigtenzahl die Chance auf Altersteilzeit höher, hier scheint die Altersteilzeit als klassisches passives Anpassungsinstrument zum Personalabbau genutzt worden zu sein. Im Osten war dagegen in Branchen mit zunehmenden bzw. konstanten Zahlen die Wahrscheinlichkeit auf Altersteilzeit größer. Hier wurde die Altersteilzeit dazu genutzt, die Personalstruktur des Betriebes über Beschäftigungsbrücken zu verändern.

Die Inanspruchnahme von Altersteilzeit ist in Regionen mit überdurchschnittlicher Arbeitslosigkeit - entsprechend den Push-Argumenten und Hypothese 12a - größer. Die höheren Odds-Ratios in
Ostdeutschland dürften auf die dort angespannte Arbeitsmarktlage zurückzuführen sein. Während hohe Vakanzraten im Westen - wie in Hypothese 13 vermutet - ein Indiz für eine geringere Wahrscheinlichkeit der Inanspruchnahme von Altersteilzeit sind, ist im Osten das Gegenteil der Fall. Die Altersteilzeit in West und Ost wird also z.T. aus recht unterschiedlichen Gründen eingesetzt.

\section{7 \\ Zusammenfassung und Ausblick}

Die Altersteilzeit hat sich im Laufe der Jahre zu einem sehr beliebten Weg für den meist vorgezogenen Übergang in die Nacherwerbsphase entwickelt. Dabei ist die Möglichkeit, Altersteilzeit nutzen zu kön- nen, nicht für alle Beschäftigten gleichermaßen gegeben. Die vorliegende Analyse zeigt, dass die Inanspruchnahme von vielfältigen Kriterien abhängt, insbesondere von den Gegebenheiten im Betrieb. PushFaktoren fallen ebenso ins Gewicht wie Pull-Faktoren. So haben Beschäftigte aus großen Unternehmen und aus Branchen mit hoher Tarifbindung höhere, Beschäftigte mit geringeren Einkommen dagegen schlechtere Chancen auf Altersteilzeit. Insgesamt verweisen die vorgestellten Befunde zur Nutzung der Altersteilzeit auf selektive, aber wenig zielgenaue Ergebnisse dieses Instruments hin - nach Berufen, nach Betriebsgröße und nach Branchen. Die Ergebnisse deuten darauf hin, dass die Altersteilzeit vor allem in Westdeutschland als Anpassungsinstrument zum Personalabbau genutzt wurde. Im Osten finden sich auch Anhaltspunkte, dass die Altersteilzeit entsprechend ihrer Zielsetzung dazu 
beigetragen hat, die Personalstruktur über Beschäftigungsbrücken zu verändern.

Wie sich die Inanspruchnahme nach Auslaufen der Fördermöglichkeit künftig entwickeln wird, hängt von der weiteren Ausgestaltung der tariflichen und betrieblichen Regelungen zur Altersteilzeit ab. So kann auch nach Ende der Fördermöglichkeit noch Altersteilzeit vereinbart werden - die Aufstockungsbeträge sind auch weiterhin steuer- und sozialversicherungsfrei. Insbesondere das Bank- und Versicherungsgewerbe, die Öffentliche Verwaltung und Teile des Verarbeitenden Gewerbes haben bereits weiterführende Regelungen ausgehandelt. Zum Teil wurden diese auf Arbeitnehmer beschränkt, die besonderen Belastungen ausgesetzt sind. Da es aufgrund unterschiedlicher gesund- heitlicher Belastungen nicht für alle Beschäftigten gleichermaßen möglich ist, bis zum gesetzlichen Ruhestandsalter in vollem Maße im gleichen Beruf erwerbstätig zu bleiben, ist es insbesondere wichtig, für diese Personengruppen Perspektiven für den Verlauf des Erwerbslebens zu eröffnen, z.B. über rechtzeitige Tätigkeitswechsel (Arlt et al. 2009). Angesichts der demografischen Entwicklung in Deutschland wird sich die Problemlage vor allem dieser Personengruppen weiter zuspitzen, und Betriebe werden es zunehmend mit alternden Belegschaften zu tun haben. Bisher bietet nur ein Fünftel der Betriebe Maßnahmen in ihren Unternehmen an, die speziell auf Ältere zugeschnitten sind, zu den meistgenannten gehört bislang - als Instrument der Frühausgliederung - die Altersteilzeit
(Bellmann et al. 2007). Deshalb gewinnen zukünftig Maßnahmen an Bedeutung, die dazu beitragen, die Beschäftigungsfähigkeit der älteren Mitarbeiter zu erhalten. So sollte die Arbeitsmarktpolitik auf längere Sicht den Fokus auf die Beschäftigung möglichst bis an die Ruhestandsgrenze legen - gerade bei einer bis zum 67. Lebensjahr verlängerten Lebensarbeitszeit. Hierzu gehört eine früh ansetzende und stetige Weiterbildung ebenso wie eine intensivierte betriebliche Gesundheitsförderung. Aber auch mittels flexibler Übergänge - seien es alternsgerechte Arbeitszeiten, wie eine „echte Altersteilzeit" mit individuell verkürzten Wochenarbeitszeiten der älteren Beschäftigten oder die Option der Teilrente - könnten Ältere länger im Erwerbsleben gehalten werden.

\section{LITERATUR}

Arlt, A./Dietz, M./Walwei, U. (2009): Besserung für Ältere am Arbeitsmarkt - Nicht alles ist Konjunktur, in: IAB-Kurzbericht 16 Arnds, P./Bonin, H. (2002): Frühverrentung in Deutschland: Ökonomische Anreize und institutionelle Strukturen, in: IZA Discussion Paper 666

Barkholdt, C. (2001): Rentenzugang und Altersteilzeit, in: Prekärer Übergang in den Ruhestand, S. 149-175

Bellmann, L./Janik, F. (2009): Betriebe und Frühverrentung: Angebote, die man nicht ablehnt, in: Zeitschrift für ArbeitsmarktForschung 4,

S. 311-324

Bellmann, L./Kistler, E./Wahse, J. (2007): Demographischer Wandel Betriebe müssen sich auf alternde Belegschaften einstellen, IAB-Kurzbericht 21

Brussig, M./Knuth, M./Wojtkowski, S. (2009): Altersteilzeit:

Zunehmend Beschäftigungsbrücke zum späteren Renteneintritt, Altersübergangs-Report 02

Deutsche Rentenversicherung Bund (DRV) (2008): Rentenversicherung in Zeitreihen 2007, DRV-Schriften 22

Deutsche Rentenversicherung Bund (DRV) (2009): Rentenzugang 2008, Statistik der Deutschen Rentenversicherung 173

Ellguth, P./Koller, B. (2000): Frühverrentung: Arbeitsmarktentlastung durch Altersteilzeit, in: IAB-Materialien 1, S. 4-5

Ellguth, P./Kohaut, S. (2008): Tarifbindung und betriebliche Interessenvertretung - aktuelle Ergebnisse aus dem IAB-Betriebspanel 2007, in: WSI-Mitteilungen 9, S. 515-519

Himmelreicher, R./Hagen, C./Clemens, W. (2008): Hat das Ausbildungsniveau einen Einfluss auf das individuelle Rentenzugangsverhalten?, RatSWD Research Note 30

Hirschenauer, F. (2007): Arbeiten bis 65 - längst noch nicht die Regel, IAB-Kurzbericht 25

Hoffmann, H. (2007): Wege in den Ruhestand, in: Deutsche Rentenversicherung $4-5$, S. $298-320$
Hoffmann, H./Hofmann, J. (2007): Rentenzugang 2007: Trendwende bei Zugängen in die Regelaltersrente?, in: RVaktuell 5/6/2008, S. 150-159 Hofmann, H. (2008): Altersteilzeit und Altersversorgung in der Metallund Elektroindustrie, in: ifo-Schnelldienst 3, S. 15-20

Höhne, A. (2007): Erwerbsminderungsrenten und medizinische Rehabilitation in Deutschland unter Berücksichtigung des Migrationshintergrunds, in: Gesundheit Berlin (Hrsg.): Dokumentation 12, bundesweiter Kongress Armut und Gesundheit „Präventionen für Gesunde Lebenswelten - ,Soziales Kapital' als Investition in Gesundheit"

Kaldybajewa, K. (2004): Rentenzugang BfA 2003: Jeder achte Altersrentner kommt aus der Altersteilzeit, in: Deutsche Angestellten Versicherung 5/6, S. 227-236

Kaldybajewa, K./Kruse, E. (2007): Altersteilzeit immer beliebter Statistische Fakten, Interpretationen und Bewertungen, in: RVaktuell 8, S. 244-253

Klammer, U./Weber, H. (2001): Flexibel in den Ruhestand? - Ergebnisse und Überlegungen zur Altersteilzeit, in: WSI-Mitteilungen 2,

S. $102-112$

Knuth, M. (2010): Altersteilzeit weiterhin fördern, aber Option für Umgestaltung eröffnen, IAQ-Standpunkte 01

Kruse, E. (2007): Empirische Ergebnisse zur Erwerbsbeteiligung älterer Versicherter anhand der Statistiken der gesetzlichen Rentenversicherung, in: Deutsche Rentenversicherung 11-12, S. 716-749

Leber, U./Wagner, A./(2007): Early and phased retirement in European companies. Establishment Survey on Working Time 2004-2005, hrsg. von der European Foundation for the Improvement of Living and Working Conditions

Lindecke, C./Voss-Dahm, D./Lehndorff, S. (2007): Altersteilzeit Erfahrungen und Diskussionen in Deutschland und anderen EU-Ländern, Arbeitspapier der Hans-Böckler-Stiftung 142

Naegele, G./Voges, W. (1989): Die beschäftigungspolitischen Auswirkungen des Vorruhestandsgesetzes, in: Sozialer Fortschritt 3, S. 52-63 
Pimpertz, J./Schäfer, H. (2009): Was kostet der vorzeitige Ausstieg aus dem Erwerbsleben?, in: iwTrends 1

Radl, J. (2006): Pfade in den Ruhestand und die Heterogenität des Renteneintrittsalters, in: Deutsche Rentenversicherung 9-10, S. 641-660

Radl, J. (2007): Individuelle Determinanten des Renteneintrittsalters, in: Zeitschrift für Soziologie 1, S. 43-64

Stegmann, M. (2008): Analysen zum Zusammenhang zwischen den Versicherungsbiografien von Ehepartnern und die Auswirkungen auf die finanzielle Situation im Alter, in: Deutsche Rentenversicherung 5, S. $435-461$

Stück, H. (2003): Gleitend in den Ruhestand? Gesetzliche, tarifliche und tatsächliche Entwicklung der Altersteilzeit, hrsg. von der Arbeitnehmerkammer Bremen

Wanger, S. (2009): Altersteilzeit - Beliebt, aber nicht zukunftsgerecht, IAB-Kurzbericht 8 